\title{
Monitoreo terapéutico de vancomicina intravenosa en una unidad de paciente crítico pediátrico
}

\author{
Rodolfo Villena, Claudio A. González, M. Elisa Nalegach, \\ Alexandra Vásquez, Marcela Villareal y Michele Drago
}

\section{Therapeutic monitoring of intravenous vancomycin in a pediatric critical care unit}

Background: In critically ill pediatric patients vancomycin distribution and elimination is altered underscoring the need for pharmacokinetic monitoring; however the therapeutic trough ranges have not been validated for children. Objective: To describe the pharmacokinetics of intravenous vancomycin in critically ill pediatric patients using plasmatic vancomycin monitoring. Methods: Retrospective, descriptive study performed in a paediatric critical care unit. Vancomycin serum levels (Cmin and Cpeak), $\mathrm{t} 1 / 2$ and Vd were determined in 1 month to 12 year old patients receiving $\geq 40 \mathrm{mg}$ per-kg-per day. Plasmatic levels were measured at therapy onset and during follow up, evaluating the proportion of trough level determinations within therapeutic range, the average trough concentration, and the Cpeak achieved. Results: A total of 65 plasmatic vancomycin monitorings were analysed in 45 patients. The average values for Ctrough, Cpeak, $t_{1 / 2}$ and $V d$ were $10.4 \mu \mathrm{g} / \mathrm{mL}, 22.7 \mu \mathrm{g} / \mathrm{mL}, 3,1$ $\mathrm{h}$ and $0.7 \mathrm{~L} / \mathrm{kg}$, respectively. An average dose of $47,1 \mathrm{mg} / \mathrm{kg} /$ day achieved initial Ctrough levels $<10 \mathrm{mg} / \mathrm{mL}$ in $60 \%$ of patients $(\mathrm{n}=27)$, between 10 and $14,9 \mu \mathrm{g} / \mathrm{mL}$ in $22,2 \%(\mathrm{n}=10)$, between 15 to $20 \mu \mathrm{g} / \mathrm{mL}$ in $4 \%$ (n: 2), and $>20 \mu \mathrm{g} / \mathrm{mL}$ in 13,3\% (n: 6). Conclusions: Vancomycin doses of $40 \mathrm{mg} / \mathrm{kg} /$ day are insufficient for critically ill paediatric patients without renal failure. A higher starting dose and monitoring of plasma levels must be considered in this population.

Key words: Vancomycin, pharmacokinetics, children, criticall illness.

Palabras clave: Vancomicina, farmacocinética, niños, paciente crítico.

\section{Introducción}

$\mathrm{V}$ ancomicina es un antimicrobiano glicopéptido con acción sobre microorganismos grampositivos, efecto logrado por su fijación a precursores de la pared bacteriana ${ }^{1}$. Su distribución en el organismo es dependiente del agua corporal y se estima con el volumen de distribución aparente $(V d)$ en $0,2-0,9 \mathrm{~L} / \mathrm{kg}^{2}$. Tiene una eliminación altamente dependiente de la función renal y estimada con el tiempo de vida media $\left(t_{1 / 2}\right)$ en 4 a $6 \mathrm{~h}$, evaluada en pacientes sin disfunción renal $^{3}$. Se ha asociado su uso con la aparición de nefrotoxicidad y ototoxicidad, aumentando este riesgo con concentraciones plasmáticas basales (Cbasal) altas, uso prolongado y presencia de otras sustancias ototóxicas o nefrotóxicas ${ }^{4}$.

El incremento sostenido de la resistencia estafilocócica a penicilina y sus derivados le ha dado un rol cada vez más vigente, sobre todo en pacientes críticos, donde las condiciones clínicas fomentan alteraciones en su función renal y en su distribución del agua corporal total, provocando amplias variaciones en las concentraciones plasmáticas de vancomicina, lo que tiene el riesgo potencial de toxicidad o de obtenerse dosis sub-terapéuticas ${ }^{5,6}$. Junto con la función renal y el peso del paciente, la edad es un determinante importante en la eliminación y distribución del fármaco ${ }^{2,6}$. En pediatría, independiente de su condición patológica o edad, la dosificación se realiza en base a recomendaciones internacionalmente aceptadas con esquemas de 40 a $60 \mathrm{mg} / \mathrm{kg} /$ día en pacientes sin disfunción renal ${ }^{7}$.

El monitoreo terapéutico de vancomicina es una herramienta práctica que permite ajustar la dosificación mediante determinación de concentraciones sanguíneas, con el propósito de obtener metas terapéuticas y reducir el riesgo de toxicidad ${ }^{8}$. Actualmente se acepta que el índice farmacocinético/farmacodinámico (FC/FD) llamado área bajo la curva $(\mathrm{ABC})$ sobre la concentración inhibitoria mínima (CIM) en $24 \mathrm{~h}\left((\mathrm{ABC} / \mathrm{CIM})_{24 \mathrm{~h}}\right)$ es el mejor predictor de eficacia y seguridad para vancomicina ${ }^{9}$. Para el cálculo del ABC se necesitan varias mediciones de concentraciones sanguíneos, lo que en la práctica no se realiza por lo que en pacientes adultos se ha estimado $\mathrm{ABC}$ utilizando Cbasales, asumiéndosele como el marcador sustituto con mejor correlación, aunque existe controversia aún sobre este punto $^{8}$. El tiempo adecuado para extraer una muestra es el instante previo a una dosis, durante el estado estacionario (alrededor de la cuarta dosis, en regímenes fraccionados cada $6 \mathrm{~h}$ ), resultado que es denominado Cbasal y que debe ser $>10 \mu \mathrm{g} / \mathrm{mL}$ para no inducir apa-
Universidad de Chile. Facultad de Medicina, Campus Sur Departamento de Pediatría (RV, AV, MV).

Hospital de Niños Dr. Exequiel González Cortés.

Unidad de Paciente Crítico (CAG, MEN, MD)).

R. Villena es asesor médico de Novartis Vacunas. Los otros autores declaran no tener conflictos de interés.

Sin financiamiento

Recibido: 16 de julio de 2013 Aceptado: 17 de abril de 2014

Correspondencia a: Rodolfo Villena Martínez rodolfo.villena@gmail.com 
rición de cepas resistentes $\mathrm{y}<20 \mu \mathrm{g} / \mathrm{mL}$ para reducir el riesgo de nefrotoxicidad ${ }^{8}$. Las concentraciones plasmáticas basales eran considerados terapéuticos si alcanzaban valores de 5 a $15 \mu \mathrm{g} / \mathrm{mL}$, lo que se lograba usualmente con las dosis pediátricas habituales. Actualmente, se acepta la recomendación entregada por la Guía de Manejo para Infecciones por Staphylococcus aureus Resistente a Meticilina en Adultos y Niños, de la Sociedad Americana de Enfermedades Infecciosas (IDSA, por sus siglas en inglés), donde se recomiendan concentraciones basales de $15-20 \mu \mathrm{g} / \mathrm{mL}$ en niños y adultos con infecciones complicadas, tales como bacteriemia, endocarditis, osteomielitis, meningitis, neumonías e infecciones graves de la piel ${ }^{10}$. Estudios en pacientes críticos pediátricos sin disfunción renal muestran que las dosis habituales de vancomicina pueden dar origen a niveles sub-terapéuticos ${ }^{11-13}$, lo que podría exponer a nuestros pacientes a fracasos clínicos, entre otras complicaciones. Este monitoreo terapéutico no está universalmente implementado para los pacientes críticos pediátricos de nuestro país, por lo que el presente estudio tiene como objetivo principal revisar y describir la experiencia de monitoreo de las concentraciones séricas de vancomicina en una institución, con el fin de determinar si las dosis recomendadas han alcanzado el rango de Cbasales deseadas para niños.

\section{Material y Métodos}

Estudio descriptivo, retrospectivo, realizado en la UPC del Hospital de Niños Dr. Exequiel González Cortés, Santiago, Chile, durante el período julio de 2010 a noviembre de 2012. El estudio fue aprobado por el Comité de Docencia e Investigación de nuestro establecimiento.

\section{Criterios de inclusión}

Se incluyeron todos los pacientes mayores de 1 mes y hasta 12 años que recibieron vancomicina en las siguientes condiciones: dosis terapéutica, fraccionada en intervalos de $6 \mathrm{~h}$, infundida durante $60 \mathrm{~min}$, monitoreados terapéuticamente según protocolo local ad hoc. Se definió como dosis terapéuticas valores sobre $40 \mathrm{mg} / \mathrm{kg} /$ día, utilizándose para su cálculo el peso total del paciente al momento de la determinación de concentraciones y la dosis administrada en relación al monitoreo (ambos datos obtenidos de los registros diarios de enfermería), aceptándose dosis de hasta $36 \mathrm{mg} / \mathrm{kg} /$ día (10\% más bajas) como terapéuticas.

\section{Criterios de exclusión}

Se excluyeron pacientes con disfunción renal, definida por al menos uno de los siguientes criterios al momento del monitoreo $^{14}$ : creatininemia $>$ límite normal para la edad; aclaramiento de creatinina estimado $<50 \mathrm{ml} /$ $\mathrm{min} / 1.73 \mathrm{~m}^{2}$, por fórmula de Schwartz; diagnóstico de in- suficiencia renal crónica; $y / o$ uso de terapias de reemplazo renal. También se excluyeron pacientes con una historia de, hasta dos semanas previas al monitoreo, incremento mayor a $50 \%$ en la creatininemia sobre el valor basal.

\section{Diseño de monitoreo terapéutico}

Vancomicina fue infundida por vía intravenosa, de manera continua, con tiempo cero, definido como el momento en que se inició la infusión. La unidad de análisis fue la monitorización sanguínea de cada paciente, definida como la toma de una concentración plasmática basal en estado estacionario, Cbasal, obtenida mínimo en torno a la cuarta dosis, con o sin una muestra para determinar concentración plasmática máxima, y Cpico, tomado en relación a la misma dosis. Se incluyeron los monitoreos con un nivel Cpico tomado media hora después de terminada la infusión y/o un Cbasal tomado media hora antes de una dosis. En cada monitoreo, el rango terapéutico para la Cbasal se estableció entre 15 y $20 \mu \mathrm{g} / \mathrm{mL}$ si presentaban bacteriemia, endocarditis, infecciones de tejidos blandos, meningitis y/o neumonía, y entre 10 y $15 \mu \mathrm{g} / \mathrm{mL}$, en otros casos $^{8}$. La unidad de información fue la ficha de monitoreo terapéutico, elaborada para cada paciente por el servicio de farmacia clínica del hospital. Los niveles plasmáticos fueron procesados con técnica de inmunoensayo de fluorescencia polarizada (Laboratorio Abbott). Los ajustes de dosis fueron realizados conjuntamente por infectólogo pediatra y farmacéutico clínico. Se evaluó el porcentaje de monitoreos con Cbasal en rango terapéutico, su promedio y distribución, así como los parámetros farmacocinéticos $\mathrm{Vd} \mathrm{y} \mathrm{t}_{1 / 2}$. Finalmente, se analizó la influencia de la edad sobre las variables mencionadas, categorizando esta variable en dos grupos: pacientes de 1 mes a $<12$ meses y pacientes entre 1 año y $<12$ años. Este estudio utilizó un modelo monocompartamental, el cual ha sido validado en pediatría, para el cálculo de parámetros farmacocinéticos desde Cpeak y Cbasal ${ }^{6}$. En los casos donde se obtuvieron ambos niveles en torno a una misma dosis se calcularon el $t_{1 / 2} \mathrm{y} V d$. Las fórmulas utilizadas fueron:

Constante de eliminación, $\mathrm{Ke}=[\ln ($ Cpico $\div$ Cbasal $)] \div$ (Tmin - Tmax)

Tiempo de vida media, $\mathrm{t}_{1 / 2}=0,693 \div \mathrm{Ke}$

$\mathrm{V}_{\mathrm{d}}=\left(\right.$ Dosis $\left.\div \mathrm{t}^{\prime}\right) \mathrm{x}\left[1-\mathrm{e}^{\left(-\operatorname{Kex} \mathrm{t}^{\prime}\right)}\right] \div\left(\right.$ Ke x $\left[\mathrm{Cpico}_{\mathrm{C}} \mathrm{e}^{(-\operatorname{Kex}}\right.$ ${ }^{\mathrm{T} \max )}-$ Cbasal $\left.\left.\mathrm{x} \mathrm{e}^{(-\mathrm{Ke} \times \mathrm{Tmin})}\right]\right)$.

Donde $\mathrm{t}^{\prime}=$ tiempo de infusión.

\section{Análisis estadístico}

Se utilizó el programa estadístico EpiInfo 2000 StatCalc para el análisis de datos. Las variables categóricas fueron expresadas como porcentaje y las variables cuantitativas como valor promedio, con su desviación estándar y/o rango de distribución. Las variables continuas fueron comparadas mediante la prueba no paramétrica de Mann- 
Whitney para muestras independientes y las cualitativas con la prueba de $\chi^{2}$-cuadrado. Se asumió significancia estadística cuando los valores de $\mathrm{p}$ en las pruebas fueron menores de 0,05 .

\section{Resultados}

Durante el período de estudio se realizaron 120 monitoreos terapéuticos de vancomicina en 70 pacientes. Se excluyeron 55 monitoreos, correspondiendo a 25 pacientes, por los siguientes motivos: presencia de disfunción renal (n: 28), sin requisito de edad (n: 10), infusiones mayores a $1 \mathrm{~h}$ (n: 15), dosis sub-terapéutica (n: 1) e intervalo distinto a $6 \mathrm{~h}$ (n: 1). Las características demográficas asociadas a los 45 pacientes que ingresaron al estudio se describen en la Tabla 1.

\section{Datos farmacocinéticos globales}

Se incluyeron 65 monitoreos terapéuticos, obteniéndose una Cbasal promedio de 10,4 $\mu \mathrm{g} / \mathrm{mL}$ (rango 1,4-25,5). En todos los casos clínicos el rango terapéutico buscado fue de 15 a $20 \mathrm{mg} / \mathrm{mL}$. En 32 monitoreos se obtuvo una Cpico, siendo su promedio de $22,7 \mu \mathrm{g} / \mathrm{mL}$ (Figura 1). El $t_{1 / 2}$ promedio se estimó en $3,1 \mathrm{~h}(1,1-7,7)$, sin diferencias significativas por grupos etarios, y el $V d$ promedio en 0,7 $\mathrm{L} / \mathrm{kg}(0,4-2,1)$. El parámetro Cbasal fue significativamente más alto en las monitorizaciones del grupo de pacientes entre 1 y 12 meses comparado con los mayores (p: 0,027) (Tabla 2). Ambos grupos de edad tenían creatininemia basal promedio de $0,2 \mathrm{mg} / \mathrm{dL}$.

\section{Datos farmacocinéticos correspondientes al monitoreo inicial}

Cuarenta y cinco de los 65 monitoreos farmacocinéticos correspondieron a determinaciones iniciales, y se realizaron en cada uno de los 45 pacientes. La dosis promedio asociada al monitoreo inicial fue de $47,1 \mathrm{mg} /$ kg/día (rango 36-75). La Cbasal promedio de estos 45 monitoreos fue de $9,8 \mu \mathrm{g} / \mathrm{mL}$ (rango 1,4-25,5). Dos niveles basales $(4,4 \%)$ alcanzaron el rango terapéutico, 10 $(22,2 \%)$ fueron entre 10 a $14,9 \mu \mathrm{g} / \mathrm{mL}, 6(13,3 \%)$ fueron sobre $20 \mu \mathrm{g} / \mathrm{mL}$ y $27(60 \%)$ bajo $10 \mu \mathrm{g} / \mathrm{mL}$.

\section{Datos farmacocinéticos de las monitorizaciones posteriores a la inicial}

Veinte de los 65 monitoreos farmacocinéticos correspondieron a determinaciones posteriores a la inicial, y se realizaron en 15 de los 45 pacientes. La Cbasal promedio de estos 20 monitoreos fue más alta que la obtenida en la monitorización inicial de los 15 pacientes, al igual que las dosis promedio utilizadas (Cbasal: 11,6 versus 7,6 $\mu \mathrm{g}$ / $\mathrm{mL}$ y dosis: 57 versus $47 \mathrm{mg} / \mathrm{kg} /$ día, respectivamente). La proporción de niveles basales sobre $20 \mu \mathrm{g} / \mathrm{mL}$ fue de $14 \%$.
Tabla 1. Características clínicas y demográficas de los pacientes críticos pediátricos incluidos en el estudio (n: 45)

\begin{tabular}{lc} 
Características & Valor* \\
Edad (años) & 2,3 (rango 1 mes a 10 años) \\
Grupo etario (\%) & 49 \\
$\quad 1$ mes a $<12$ meses & 51 \\
1 año a $<12$ años & 57 \\
Sexo masculino (\%) & 11,7 (rango 2,6-52) \\
Peso (kg) & 0,2 (rango 0,1-0,4) \\
Creatininemia (mg/dL) & 4 \\
Co-morbilidades ( $n$ de pacientes) & 5 \\
Cáncer & 1 \\
Gran quemado & \\
Fibrosis quística & \\
\hline *Las variables cuantitativas se expresan como valor promedio y en \\
paréntesis el rango.
\end{tabular}

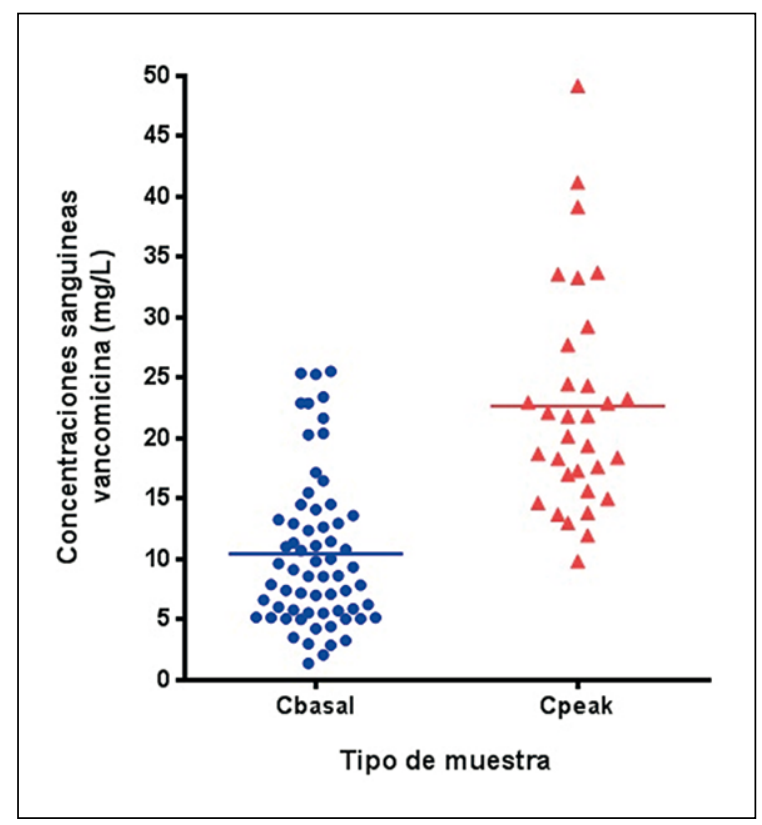

Figura 1. Distribución de todas las concentraciones plasmáticas Cbasal y Cpico para vancomicina en pacientes críticos pediátricos (El promedio es representado en una barra horizontal). 


\section{Discusión}

El utilizar concentraciones plasmáticas de vancomicina nos permite ajustar su dosificación con el propósito de alcanzar las metas terapéuticas y reducir su riesgo de toxicidad ${ }^{8}$. La práctica habitual del monitoreo se realiza con un Cbasal en estado estacionario. No obstante ello, existen pocos reportes científicos en relación a su uso en pediatría. Los nuevos valores de concentración plasmática mínima, que rigen a partir del año 2009, son más altos que los establecidos previamente ${ }^{10}$, lo que se sustenta en la optimización de la eficacia y reducción de la resistencia, a través de la obtención de índices FC/FD adecuados ${ }^{15}$. Para vancomicina, el índice $\left((\mathrm{ABC} / \mathrm{CIM})_{24} \mathrm{~h}\right)$, parece ser el mejor predictor de eficacia en modelos animales y humanos ${ }^{16}$, aceptándose como adecuado un valor sobre $400^{8,17}$, valor que en adultos se lograría si las Cbasales se sitúan entre 15 y $20 \mu \mathrm{g} / \mathrm{mL}$ para infecciones por $S$. aureus con CIM de $1 \mathrm{mg} / \mathrm{ml}^{18}$. En la población pediátrica se asume igual relación entre Cbasal y ABC/CIM que los adultos; sin embargo, carecemos de evidencia clara que lo respalde ${ }^{19}$. Este estudio no evaluó la capacidad de los tratamientos de alcanzar valores del índice FC/FD área bajo la curva $\left((\mathrm{ABC} / \mathrm{CIM})_{24 \mathrm{~h}}\right)$ mayores a 400 y si tomáramos en consideración el parámetro recomendado por las guías de monitoreo terapéutico de la IDSA, la mayor parte de nuestros pacientes pediátricos no alcanzarían las concentraciones plasmáticas actualmente sugeridas. En vista de estos resultados, parece adecuado aumentar las dosis iniciales o reformular la Cbasal necesaria para que estos grupos etarios alcancen valores de $\mathrm{ABC} / \mathrm{CIM}$ recomendados ${ }^{20}$.

Nuestros resultados concuerdan con lo descrito por otros autores ${ }^{11-13}$, quienes demuestran que se obtienen valores promedios de Cbasal menores que $10 \mathrm{mg} / \mathrm{mL}$ al realizar el monitoreo inicial con dosis de $40 \mathrm{mg} / \mathrm{kg} /$ día, lográndose superar esta concentración tras un aumento en la dosificación, sin que ello signifique un incremento en la proporción de concentraciones en rango tóxico, lo que justificaría comenzar con dosis mayores a $40 \mathrm{mg} / \mathrm{kg} /$ día, sobre todo en pacientes de 1 a 12 años, realizando monitoreo terapéutico de niveles séricos para eventuales ajustes. De este modo, de manera concordante con otros trabajos, pareciera que la condición pediátrica es un factor de riesgo per se para obtener niveles plasmáticos de vancomicina sub-terapéuticos ${ }^{21,22}$, lo que, adicionado a la condición de patología crítica que cursan estos pacientes, hace que se expliquen los hallazgos de nuestro trabajo, aún en ausencia de disfunción renal, lo que podría deberse a las diferencias de maduración de órganos eliminadores, como el riñón. Estas diferencias farmacocinéticas podrían justificar el utilizar dosis iniciales diferentes para cada grupo de edad, pero se necesitan más estudios para establecer dicha recomendación.
Este estudio fue diseñado para observar el comportamiento de los niveles plasmáticos de vancomicina en pacientes críticos sin disfunción renal; sin embargo, esta disfunción es prevalente durante la evolución de pacientes en unidades de cuidado intensivo pediátrico ${ }^{23}$. Por lo mismo, la necesidad de ajuste de dosis y el riesgo de toxicidad de este antimicrobiano cobra mayor relevancia en estas situaciones y con ello, la necesidad de su monitoreo terapéutico ${ }^{24}$.

La vancomicina utilizada durante este estudio correspondió a las producidas por los laboratorios Hospira y TEVA, sin ser uno de nuestros objetivos el analizar la influencia de las distintas marcas sobre la capacidad de alcanzar niveles plasmáticos adecuados.

Es importante destacar que este estudio presenta importantes limitaciones como su carácter retrospectivo, la no medición de variables confundentes como presencia de otros fármacos nefrotóxicos, fármacos que competirían por los mecanismos de secreción y la limitación propia de la técnica de medición ${ }^{25}$. Adicionalmente, el hecho de ocupar un modelo monocompartimental a partir de dos concentraciones plasmáticas para determinar parámetros farmacocinéticos, utilizar datos obtenidos en relación a administraciones estandarizadas para infusión y fraccionamiento, podrían representar estimaciones menos precisas que la utilización de otras técnicas más complejas ${ }^{6}$.

En conclusión, nuestro estudio demuestra que las dosis habituales de vancomicina, administradas en infusión de una hora y a intervalos de seis horas son insuficientes para los pacientes pediátricos críticos sin disfunción renal, por lo que parece adecuado, en estos pacientes, comenzar con dosis mayores, sobre todo en pacientes entre 1 y 12 años. La amplia variabilidad observada en la Cbasal y la baja proporción de niveles en rango terapéutico justifica el monitoreo terapéutico como herramienta terapéutica en el manejo de los pacientes críticos pediátricos sin disfunción renal.

Agradecimientos: Al personal médico y de enfermería de la Unidad de Paciente Crítico por su valiosa labor en la implementación del monitoreo terapéutico de vancomicina en nuestro hospital.

\section{Resumen}

Introducción: Los pacientes críticos pediátricos presentan alteraciones en la distribución y eliminación de vancomicina, lo que hace necesaria su monitorización terapéutica; sin embargo, los rangos basales óptimos no han sido validados en niños. Objetivo: Describir el monitoreo terapéutico de vancomicina intravenosa en pacientes críticos pediátricos, a través de medición de 
concentraciones plasmáticas terapéuticas. Metodología: Estudio descriptivo, retrospectivo, en una Unidad de Paciente Crítico Pediátrica. Se analizaron concentraciones plasmáticas de vancomicina Cbasales y Cpico, en niños entre 1 mes y 12 años de edad, que recibieron dosis $\geq 40$ $\mathrm{mg} / \mathrm{kg} /$ día. Se registraron concentraciones plasmáticas iniciales y de seguimiento, evaluándose la proporción de concentraciones sanguíneas basales en rango terapéutico, la concentración basal promedio y el Cpeak alcanzado. Resultados. Se analizaron 65 monitoreos terapéuticos, correspondientes a 45 pacientes. Los valores promedio de
Cbasal, Cpico, $t_{1 / 2 y} V d$ fueron $10,4 \mu \mathrm{g} / \mathrm{mL}, 22,7 \mu \mathrm{g} / \mathrm{mL}, 3,1$ h y $0,7 \mathrm{~L} / \mathrm{kg}$, respectivamente. Las Cbasales iniciales de los 45 pacientes, usando dosis promedio de $47,1 \mathrm{mg} / \mathrm{kg} /$ día, se encontraron en $60 \%$ (n: 27 ) de los casos $<10 \mu \mathrm{g} /$ $\mathrm{mL}$, entre 10 y $14,9 \mu \mathrm{g} / \mathrm{mL}$ en $22 \%$ (n: 10 ), en $46 \%$ entre 15 y $20 \mu \mathrm{g} / \mathrm{mL}$ (n: 2) y en 13,3\% (n: 6) fueron $>20 \mu \mathrm{g} /$ $\mathrm{mL}$. Conclusión: Vancomicina en dosis de $40 \mathrm{mg} / \mathrm{kg} /$ día, es insuficiente para pacientes pediátricos críticos sin disfunción renal, por lo que parece recomendable comenzar con dosis mayores y realizar monitoreo terapéutico de concentraciones plasmáticas en estos casos.

\section{Referencias bibliográficas}

1.- Jones R N. Microbiological features of vancomycin in the $21^{\text {st }}$ Century: minimum inhibitory concentration creep, bactericidal/ static activity, and applied breakpoints to predict clinical outcomes or detect resistant strains. Clin Infect Dis 2006; 42 Suppl 1: S13-24.

2.- Marsot A, Boulamery A, Bruguerolle B, Simon N. Vancomycin: a review of population pharmacokinetic analyses. Clin Pharmacokinet. 2012; 51 (1): 1-13.

3.- Cunha B A. Vancomycin. Med Clin North Am 1995; 79 (4): 817-31.

4.- Lodise T P, Patel N, Lomaestro B M, Rodvold K A, Drusano G L. Relationship between initial vancomycin concentration-time profile and nephrotoxicity among hospitalized patients. Clin Infect Dis 2009; 49 (4): 507-14.

5.- Fridkin S K, Edwards J R, Pichette S C, Pryor E R, McGowan J E Jr, Tenover F C, et al. Determinants of vancomycin use in adult intensive care units in 41 United States hospitals. Clin Infect Dis 1999; 28 (5): 1119-25.

6.- Roberts J A, Lipman J. Pharmacokinetic issues for antibiotics in the critically ill patient. Crit Care Med 2009; 37 (3): 840-51.

7.- Rodvold K A, Everett J A, Pryka R D, Kraus D M. Pharmacokinetics and administration regimens of vancomycin in neonates, infants and children. Clin Pharmacokinet 1997; 33 (1): 32-51.

8.- Rybak M, Lomaestro B, Rotschafer JC, Moellering R Jr, Craig W, Billeter M, et al. Therapeutic monitoring of vancomycin in adult patients: a consensus review of the American Society of Health-System Pharmacists, the Infectious Diseases Society of America, and the Society of Infectious Diseases Pharmacists. Am J Health Syst Pharm 2009; 66 (1): 82-98.

9.- Giachetto G A, Telechea H M, Speranza N,
Oyarzún M, Nanni L, Menchaca A. Vancomycin pharmacokinetic-pharmacodynamic parameters to optimize dosage administration in critically ill children. Pediatr Crit Care Med 2011; 12 (6): e250-4.

10.- Liu C, Bayer A, Cosgrove S E, Daum R S, Fridkin S K, Gorwitz R J, et al. Clinical practice guidelines by the Infectious Diseases Society of America for the treatment of methicillin resistant Staphylococcus aureus infections in adults and children. Clin Infect Dis 2011; 52 (3): 285-92.

11.- Glover M L, Cole E, Wolfsdorf J. Vancomycin dosage requirements among pediatric intensive care unit patients with normal renal function. $\mathrm{J}$ Crit Care 2000; 15 (1): 1-4.

12.- Zylbersztajn B L, Travaglianti M, Weller G, Mato H G. Therapeutic monitoring of vancomycin in critical care pediatric patients. [Article in Spanish]. Arch Argent Pediatr 2008; 106 (1): 26-31.

13.- Khotaei G T, Jam S, Seyed Alinaghi S, Motamed F, Nejat F, Taghi M, et al. Monitoring of serum vancomycin concentrations in pediatric patients with normal renal function. Acta Med Iran 2010; 48 (2): 91-4.

14.- Minejima E, Choi J, Beringer P, Lou M, Tse E, Wong-Beringer A. Applying new diagnostic criteria for acute kidney injury to facilitate early identification of nephrotoxicity in vancomycintreated patients. Antimicrob Agents Chemother 2011; 55 (7): 3278-83.

15.- Kullar R, Davis S L, Levine D P, Rybak M J. Impact of vancomycin exposure on outcomes in patients with methicillin-resistant Staphylococcus aureus bacteremia: support for consensus guidelines suggested targets. Clin Infect Dis 2011; 52 (8): 975-81.

16.- Craig W A. Basic pharmacodynamics of antibacterials with clinical applications to the use of beta-lactams, glycopeptides, and linezolid. Infect Dis Clin North Am 2003; 17 :
479-501.

17.- Rybak M J. The pharmacokinetic and pharmacodynamic properties of vancomycin. Clin Infect Dis 2006; 42 Suppl 1: S35-9.

18.- Moise-Broder P A, Forrest A, Birmingham M C, Schentag J J. Pharmacodynamics of vancomycin and other antimicrobials in patients with Staphylococcus aureus lower respiratory tract infections. Clin Pharmacokinet 2004; 43: 925-42.

19.- Gordon C L, Thompson C, Carapetis J R, Turnidge J, Kilburn C, Currie B J. Trough concentrations of vancomycin: adult therapeutic targets are not appropriate for children. Pediatr Infect Dis J 2012; 31 (12): 1269-71.

20.- Le J, Bradley J S, Murray W, Romanowski G L, Tran T T, Nguyen N, et al. Improved vancomycin dosing in children using area under the curve exposure. Pediatr Infect Dis J 2013; 32 (4): e155-63.

21.- Eiland L S, English T M, Eiland E H 3rd. Assessment of vancomycin dosing and subsequent serum concentrations in pediatric patients. Ann Pharmacother 2011; 45 (5): 582-9.

22.- Broome L, So T Y. An evaluation of initial vancomycin dosing in infants, children, and adolescents. Int J Pediatr 2011; 2011: 470364

23.- Macleod S. Therapeutic drug monitoring in pediatrics: how do children differ? Ther Drug Monit 2010; 32 (3): 253-6.

24.- Merouani A, Flechelles O, Jouvet P. Acute kidney injury in children. Minerva Pediatr 2012; 64 (2): 121-33.

25.- Vandecasteele S J, De Vriese A S. Vancomycin dosing in patients on intermittent hemodialysis. Semin Dial 2011; 24 (1): 50-5.

26.- Anne L, Hu M, Chan K, Colin L, Gottwald K. Potential problem with fluorescence polarization immunoassay cross-reactivity to vancomycin degradation product CDP-1: its detection in sera of renally impaired patients. Ther Drug Monit 1989; 11 (5): 585-91. 